\title{
Le Delta du Danube, entre enjeux socio-économiques et préservation des ressources naturelles : exemple d'une « double transition ». Revue Pour, 217.
}

Résumé : La mise en place de la Réserve de Biosphère du Delta du Danube, tout de suite après la chute du régime communiste, place cette région si particulière en Europe, dans un processus de transition très complexe, comprenant des dynamiques socio-économiques et écologiques interdépendantes et complexes. Dans cet article, nous montrons comment la mise en place d'une réserve naturelle a été l'élément central de cette transition, à travers les reconfigurations institutionnelles et territoriales dont elle a été porteuse. Cette nouvelle structure de gestion se voie face aux difficultés d'harmonisation de l'équilibre écologique de la région avec les besoins de développement local.

\section{$\underline{\text { La reconnaissance du caractère écologiquement remarquable du Delta du Danube }}$}

Situé dans la région de Dobroudja au sud-est de la Roumanie, le delta du Danube est une vaste zone humide qui abrite une biodiversité écologique remarquable ${ }^{1}$ pour le continent Européen. Avec $82 \%$ de sa superficie totale $\left(4178 \mathrm{~km}^{2}\right)$ situés sur le territoire de la Roumanie et $18 \%$ en Ukraine, le delta est une région frontalière (cf. image 1), avec des marges sociopolitiques et culturelles extrêmement fluides (Mitroi, Beaumont, 2011). Son territoire comprend de nombreux lacs d'eau douce reliés entre eux par d'étroits chenaux couverts de végétation aquatique dense. L'eau couvre toujours $80-90 \%$ de la surface totale du delta, avec un impact direct sur les possibilités de développement économique et social dans la région. Malgré les importants travaux ${ }^{2}$ d'aménagement réalisés sur le territoire du delta du Danube pendant le régime communiste, la région est reconnue dans les documents officiels comme le delta le «plus naturel »d'Europe (UNESCO / UICN, 1991), englobant la plus grande zone marécageuse d'un seul tenant du continent. La valeur écologique de cette région est reconnue par l'Etat roumain dès les années 1930 mais les espaces protégés institués ${ }^{3}$ dans le delta à cette époque demeurent, pendant les 45 ans de régime socialiste, des « réserves sur papier », sans aucun pouvoir réglementaire.

Dans les années 1960, se pose ouvertement la question de la contribution du delta à l'édification du communisme. Le régime d'exploitation économique intensif et hypercentraliséé qui caractérise la période socialiste prônait le «contrôle total » des «forces productives » de la société et des milieux naturels, dont la «productivité naturelle » était considérée comme insuffisante par rapport aux ambition du système socialiste. Selon le premier «Plan d'Aménagement Complexe du Delta » formulé en 1969, la région est partagée en fonction des spécificités hydrographiques des espaces deltaïques: les terrains hauts sont attribués à l'agriculture, ceux moins hauts à la «pisciculture dirigée », à l'élevage ou à l'exploitation du roseau et ceux situés sous le niveau de la mer sont réservés exclusivement au secteur piscicole. Ce plan initial va évoluer vers un contrôle de plus en plus important de la fonctionnalité des terrains, car c'est l'agriculture qui devrait devenir le secteur d'activité dominant, secondé l'extension des

\footnotetext{
${ }^{1}$ La réserve de la biosphère du delta du Danube avec une superficie de $5800 \mathrm{~km}^{2}$, incluant le delta du Danube et les lagunes connexes longeant la mer Noire, est la plus importante zone humide européenne, source de 32 écosystèmes différents, 1668 espèces de plantes, 3864 espèces de faune sauvage, dont 160 espèces de poissons et 325 espèces d'oiseaux. Les seuls deltas européens comparables sont : le delta du Rhône (qui est fortement artificialisé) et celui du Guadalquivir (qui représente seulement un douzième de la surface du delta du Danube).

${ }^{2}$ A la fin de l'année 1989, 100000 ha, soit $15 \%$ du territoire du delta était sortis du circuit naturel ou directement modifié par l'intervention humaine (Gastescu, 1996).

${ }^{3}$ Dans le delta du Danube, la Forêt Letea est déclarée par le Conseil des Ministres en 1938 la première réserve naturelle de Roumanie, mondialement reconnue par l'UNESCO dans les années 70. 
aménagements piscicoles et sylvicoles. Pour coordonner cette exploitation complexe, l'État va mettre toutes les activités économiques de la région sous la subordination d'une entreprise d'État : La Centrale du delta du Danube, chargée avec l'aménagement et l'exploitation intégrale du delta. Le régime communiste laisse comme héritage au delta une structure administrative hypercentralisée, des aménagements agricoles et piscicoles improductifs (Gastescu, 1996, 2006) et une économie locale dépendante de l'exploitation des ressources naturelles.

La mise en place d'une Réserve de Biosphère en septembre 1990, quelques mois seulement après la chute du système communiste en Roumanie est un processus complexe, qui ne peut pas être compris en dehors du contexte de transition socio-économique profonde qui traverse la société roumaine au début des années 1990. Bien que l'UNESCO reconnaît ${ }^{4}$ l'importance écologique du delta en 1991, la transformation du delta d'une « unité de production » en «écosystème naturel » ne se produit pas de manière automatique. La mise en place d'une administration propre pour la région (l'ARBDD - Administration de la Réserve de Biosphère du Delta du Danube) représente un défi de création institutionnelle d'un nouveau modèle de coordination des acteurs et de leurs actions vis-à-vis de l'environnement et des ressources du territoire. Le changement du système socio-économique (privatisation et démantèlement de la Centrale, décentralisation, etc.) s'est progressivement accompagné d'une «transition écologique », par l'introduction de politiques de conservation. Cette double transition présente des aspects contradictoires et conflictuels, dont nous présentons seulement les deux plus marquants : selon une dimension territoriale et une dimension institutionnelle.

La reconfiguration territoriale du delta après 1990 : espaces naturels vs. espaces sociaux

La territorialisation (Di Meo, 1991; Fall, 2005; Déverre, 2004) dont est porteuse la Réserve de Biosphère a représenté un chantier majeur de redéfinition des relations entre les acteurs de la réserve (ARBDD, villages de la réserve et villages limitrophes, Etat, investisseurs privés, etc.) car elle a un impact direct sur la définition des politiques et leur mise en pratique. Nous considérons deux dimension de cette territorialisation de la réserve du Delta du Danube : une globale, correspondant au modèle d'organisation d'une réserve de biosphère, une autre «locale », correspondant aux spécificités de l'organisation sociopolitique de la région. Ces deux logiques contradictoires qui se superposent dans le fonctionnement d'une réserve, nécessitent un effort supplémentaire d'intégration : une logique d'isolement de la nature de la société par la création de zones strictement protégées et une logique d'intégration de la nature avec les stratégies des acteurs à propos des éléments de la Nature (ressources naturelles, paysages, etc.).

La dimension globale est incarnée par le modèle d'organisation d'une réserve en trois zones fonctionnelles de protection, une condition sine qua non pour la reconnaissance d'une réserve de biosphère, selon les critères institués par l'UNESCO. Nous retrouvons ainsi dans le delta du Danube, une aire centrale constituée par les «zones strictement protégées » (en nombre de 18), des «zones tampons » et une zone de transition ou «zones d'activités économiques » (cf. image 2). La délimitation fonctionnelle des zones de protection a été réalisée par rapport à l'importance des espèces et des écosystèmes existants sur le territoire, mais aussi par rapport à l'empreinte de la société (la présence et le degré d'intervention humaine). Une vocation spatiale de la nature est donc mobilisée en distinguant «l'environnement social » de «l'environnement

\footnotetext{
${ }^{4}$ World Heritage Nomination - IUCN Summary. Prepared by IUCN (April 1991), based on the original nomination and summary submitted by the Government of Romania.
} 
naturel ». Autrement dit, la nature se trouve dans les territoires où la société ne l'est pas. A cette dimension spatiale, s'ajoute une dimension temporaire : là où l'impact humain a été minimal, la réserve va essayer de le maintenir ainsi, en réglementant très strictement l'accès.

La dimension locale de la territorialisation est représentée principalement par le régime foncier de la réserve, où les différentes divisions territoriales sont placées sous le contrôle des institutions et des acteurs. Ce qui est séparé par les zones fonctionnelles (nature et société) est rassemblé ainsi par la conception de la propriété foncière et du système de droits (Ost, 2005). Si le modèle fonctionnel est imposé par les institutions internationales (UNESCO), la délimitation foncière tient de la décision de chaque Etat qui décide ainsi les logiques de gestion à mettre en place. Dans le delta du Danube, au moment de la création de la réserve, la propriété privée, soit elle foncière ou des infrastructures de production, est pratiquement inexistante. La question de la propriété des terrains productifs (polders) crées par le régime socialiste et de l'infrastructure de la Centrale sera également posée à l'intérieur de la réserve. Après le Décret gouvernemental ${ }^{5}$ qui mettait un terme aux travaux de transformation des milieux et arrêtait pratiquement tout investissement productif dans la région, le patrimoine de la Centrale (avec toutes ses unités économiques et terrains productifs) est transféré du ministère de l'Agriculture vers la Préfecture de Tulcea. Cet acte qui peut être jugé comme une forme de décentralisation, fait «disparaître » la structure «coupable » pendant la période socialiste de la destruction des milieux naturels, tout en gardant le contrôle étatique sur une grande partie de la région à travers l'institution de la réserve ${ }^{6}$. Tous les domaines terrestres et aquatiques du delta sont d'abord définis comme «patrimoine naturel, domaine d'intérêt national » ${ }^{7}$ et placés sous tutelle de l'ARBDD. Quelques « exceptions » à cette règle sont ensuite reconnues : les terrains qui se trouvent en propriété privée, les terrains appartenant aux unités administratives territoriales locales et les aménagements agricoles et piscicoles (qui entrent en propriété de la région). Le reste du territoire, autrement dit, les terrains qui «n'appartiennent à personne » et sans implantation d'activité économique de production sont placés sous l'administration directe de la réserve (cf. tableau 1).

Tableau 1. La territorialisation du delta du Danube

\begin{tabular}{|l|l|l|l|l|}
\hline Type de zone & $\begin{array}{l}\text { Zones } \\
\text { strictement } \\
\text { protégées } \\
(8,7 \%)\end{array}$ & $\begin{array}{l}\text { Zones tampons } \\
(38,5 \%)\end{array}$ & \multicolumn{2}{|l|}{ Zones économiques (52,8\%) } \\
\hline $\begin{array}{l}\text { Type de } \\
\text { propriété }\end{array}$ & $\begin{array}{l}\text { Domaine public d'intérêt national } \\
81,83 \%\end{array}$ & $\begin{array}{l}\text { Domaine public } \\
\text { d'intérêt régional } \\
12,29 \%\end{array}$ & $\begin{array}{l}\text { Propriété communale et } \\
\text { privée } 5,05 \%\end{array}$ \\
\hline $\begin{array}{l}\text { Catégorie de } \\
\text { bien }\end{array}$ & Eaux et terrains en régime naturel & $\begin{array}{l}\text { Polders piscicoles et } \\
\text { agricoles }\end{array}$ & $\begin{array}{l}\text { Foyers des villages, } \\
\text { propriété privée, pâturages } \\
\text { et lacs communaux }\end{array}$ \\
\hline Acteurs & $\begin{array}{l}\text { Le gouvernement à travers } \\
\text { l'administration de la réserve }\end{array}$ & $\begin{array}{l}\text { Conseil régional } \\
\text { Entreprises privées }\end{array}$ & $\begin{array}{l}\text { Les administrations locales } \\
\text { et les personnes privées }\end{array}$ \\
\hline
\end{tabular}

\footnotetext{
${ }^{5}$ Décret $\mathrm{n}^{\circ} 103$ relatif à l'abolition des travaux dans le delta du Danube.

${ }^{6}$ L'ARBDD est directement suborbonée au ministère de l'Environnement et son gouverneur et nommé directement par le Premier Ministre.

7 «Tous les domaines terrestres et aquatiques y compris les terrains situés en permanence sous l'eau, qui se trouvent dans le périmètre de la réserve, ainsi que les ressources naturelles qu'ils génèrent, constituent le patrimoine naturel, domaine d'intérêt national, et entrent dans l'administration de l'ARBDD» (loi nr. 82/1993, art. 10). 
Le résultat foncier de cette logique est que seulement un peu plus de 5\% du territoire de la réserve est considéré comme propriété d'intérêt local. En échange, $81 \%$ du territoire est placé sous l'administration de la réserve, et donc de l'Etat, beaucoup plus que les $8 \%$ compris dans les zones strictement protégées. Cette situation est dénoncée dans les années 1990, par des représentants ${ }^{8}$ des communautés locales qui considèrent qu'à travers l'ARBDD s'est instauré un régime de propriété publique de l'État sur la quasi-totalité du delta, sans prendre en compte les besoins économiques des communautés locales dépendantes des ressources naturelles, qualifiées désormais comme "publiques ». Cette impression de no man's land peut créer l'illusion d'une nature sans enjeu majeur le développement des communautés locales, alors que justement l'économie locale (agriculture, pêche, tourisme) y est dépendante. Dans cette nouvelle reconfiguration institutionnelle, les communautés locales sont vite apparues comme «problématiques ». La centralité des décisions dans la création de la réserve et le manque de consultation des communautés locales (voir : l'absence des élus ou de représentants locaux dans le Conseil Administratif de la réserve) donnent aux habitants le sentiment qu'ils ont été « placés » dans une réserve qui ne représente pas leurs intérêts.

L'administration de la réserve se voit elle, attribuer le rôle complexe d'assurer à la fois l'introduction des mesures de réglementation plus strictes d'accès aux ressources naturelles (principalement piscicoles), mais aussi de garantir la continuité des «activités économiques traditionnelles », dont la pêche fait figure emblématique, et même des «mesures de protection sociale $»^{9}$ pour les habitants de la réserve. Dans le système juridique, ce droit coutumier est inscrit comme «droit de préemption » spécifiant la priorité donnée à la population locale dans la pratique de la pêche et comme droit à la pêche familiale. Les espaces de pêche évoluent à l'intérieur de la réserve, en fonction des différentes fonctions qui leurs sont attribuées à un moment donné par gestionnaires et usagers. L'impact de la réserve sur les zones de pêche conduit à une diversification des espaces en fonction des enjeux et des acteurs qui entrent en scène : des espaces définis par l'enjeu écologique (les zones de protection intégrale et zones tampons où la pêche est interdite), des espaces de production(zone de pêche, polders piscicoles), des espaces sociaux (pour la pêche familiale), des espaces de loisir (pêche amateur).

$\underline{\text { Reconfiguration institutionnelle du delta : logique économique vs. logique écologique }}$

Dans le contexte de libéralisation des activités économiques, nous assistons dans le delta au début des années 1990, comme dans toute la société roumaine, à l'ouverture au monde de toute une société avec ses aspirations au développement, aux droits et aux libertés individuelles. Cette logique a quelques points d'antagonisme avec les politiques de conservation de la biodiversité, qui exigent la protection des milieux naturels de tout usage excessif, en imposant des restrictions d'accès aux ressources naturelles. Les difficultés d'intégration de ces deux logiques sont particulièrement visibles dans l'évolution de la gestion des droits de pêche dans la réserve.

Historiquement, la population du delta du Danube a fluctué entre 13000 et 20000 habitants, elle est aujourd'hui estimée à un peu moins de 15000 habitants. Malgré la création de

\footnotetext{
${ }^{8}$ Le parlementaire écologiste, Echim Andrei, représentant de la minorité ukrainienne en Roumanie, lors des interventions au Parlement roumain durant les premières années d'existence de la réserve.

${ }^{9}$ Les Ordonnances Gouvernementales : 547/1994, 395/1996 et 27/1996 prévoient des tarifs réduits pour le transport naval des personnes et des marchandises de stricte nécessité, la subvention de $50 \%$ de la taxe locale pour la consommation d'électricité et d'eau potable des ménages fournie par le réseau public. Le droit à la pêche (pêche familiale) ou encore à la récolte de roseau et de jonc pour les besoins de consommation familiale est reconnu ainsi par loi pour tous les ménages habitant la réserve. 
nouveaux «villages colonies ${ }^{10}$ » sous le régime communiste et la systématisation de nombreux villages pour contribuer à «l'amélioration foncière » et à l'exploitation complexe du delta, sa population connaît dès les années 1970-1980 une régression qui se poursuit aujourd'hui encore. La fermeture progressive des entreprises communistes, peu de temps après la chute du régime socialiste, conduit à un chômage régional de jusqu'à $21 \%$ au début des années 2000, avec la pêche comme activité de secours pour les ménages en difficulté. La pisciculture connaît une baisse très importante ${ }^{11}$, se pratiquant de façon exceptionnelle, l'agriculture (là où elle est possible) reste une activité de subsistance et le tourisme, malgré son essor très spectaculaire ces derniers années, n'est pas à la porté de tous les habitants, mais plutôt une opportunité pour les plus aisés et les investisseurs extérieurs. Les programmes de reconstruction écologique, en retirant du circuit économique une partie des terrains agricoles et piscicoles, sont aussi une manière de revenir à une économie plus «traditionnelle» (en utilisant des moyens de production artisanale), redonne également à la pêche une place d'activité centrale dans le delta. Mais, la baisse de la productivité des pêcheries du delta, suite aux conséquences de l'intensification de l'exploitation économique durant le communisme (pollution et poldérisation), questionne la capacité de la pêche à subvenir aux besoins économiques de la population locale.

Dans ce contexte, malgré la baisse constante des captures enregistrées depuis 1990 (ARBDD, 2000, 2006, 2007), la pêche s'impose comme un élément central de la nouvelle organisation spatiale, économique et sociale de la réserve. La pêche est redéfinie dans le nouveau contexte de la réserve, comme l'activité économique locale «traditionnelle », tout en étant reconnue comme «moins nuisible » pour les milieux naturels que l'agriculture et la pisciculture, si les règles de régulation de l'effort de pêche sont respectées. Autrement dit, quand le delta devient « naturelle », la pêche doit devenir «traditionnelle », pour faciliter l'intégration des économies locales avec les contraintes écologiques dans le périmètre de la réserve. La devise d'action de la réserve : «Protégez la nature, conservez les traditions! » illustre bien cette vision des activités locales devenues «traditionnelles », que la réserve s'engage à préserver et garantir, comme partie intégrante du paysage deltaïque et est donc un élément central de la patrimonialisation de la région.

Les incohérences institutionnelles et l'instabilité du cadre politique et législatif (la politique de la pêche a connu des changements importants à plusieurs reprises :1997, 2002, 2005, 2007, 2009, 2010) rendent extrêmement difficile la mise en place de stratégies à long terme, autant pour le gestionnaire que pour les pêcheurs, pour l'intégration des fonctions «écologiques » et des fonctions «sociales» de la pêche. Pendant les 20 années de transition socio-écologique, le système de gestion de la pêche évolue d'un système d'accès libre basé sur des permis de pêche individuels (entre 1997 et 2005) à une forme de privatisation «totale » pendant les concessions (entre 2005 et 2007), pour finalement tenter à partir de 2008 une gestion plus «participative » par la création des associations de pêche. L'action des associations de pêcheurs reste pourtant limitée par des mécanismes structurels d'accès aux marchés et par des procédures et outils de gestion (permis et quotas de pêche) peu en clin avec les réalités locales, où économie formelle et informelle s'entremêlent de façon très complexe et rend très incertains les outils de gestion.

\footnotetext{
${ }^{10}$ Comme le village de Maliuc, situé sur le bras de Sulina à $27 \mathrm{~km}$ de Tulcea, qui a été créé en 1950 comme un centre de coordination d'exploitation du roseau et devenu «colonie des travailleurs» avec des bâtiments de bureau, des logements pour les employés. En 1956, la station est déclarée village et d'autres activités comme la pêche ont fait leur apparition. D'autres colonies de travailleurs (Pardina ou Cardon) sont aujourd'hui abandonnées à leur sort.

${ }^{11}$ Après 1990, l'activité de pisciculture va se réduire à 70 - $75 \%$ des surfaces aménagées auparavant dans ce but. 
Alors qu'on assiste à une folklorisation des traditions locales, en lien avec le développement touristique de la région, l'amalgame trop facile entre «traditionnel » et «écologique », ne répond pas aux questions liées à la définition de la pêche artisanale selon les outils de régulation (principalement droits de pêche). Dans cette évolution de la réglementation, la réserve oscille entre un rôle de définition des règles écologiques (atomisation d'environnement pour les activités économiques, évaluation et restauration des mieux naturels, etc.) et un rôle plus affirmé de régulation des activités économiques (gestionnaire des permis et quotas de pêche). Les pêcheurs car à eux, se trouvent dans une position ambiguë, à la fois victimes et causes de la dégradation, ou encore responsables directs (via les associations) de la gestion et l'amélioration des ressources.

\section{La transition dans la réserve. Comment revoir ces principes fondateurs ?}

La réserve se constituait au début des années 1990 plus comme une solution de « remplacement» de l'ancienne structure administrative de la Centrale que comme une structure de réconciliation des divers intérêts des acteurs locaux présents dans ces territoires. Pour circonscrire les activités économiques sur son territoire, la réserve introduit de nouvelles dichotomies territoriales et institutionnelles (naturel / social et économique, public / privé, national / régional / local) qui vont reformuler les relations de pouvoir dans le territoire et vont rendre possible le transfert de la responsabilité étatique pour la dégradation de l'environnement vers les activités économiques locales, principalement la pêche. Par conséquence, c'est au niveau local que les efforts d'intégration de l'économie et de la nature par le respect des exigences écologiques doivent être réalisés. Mais les séparations territoriales et institutionnelles entre «espace naturel » et «espace social» à l'intérieur de la réserve, sont loin d'avoir résolu la situation des communautés situées sur le territoire de la réserve.

Le discours de re-naturalisation des ressources et des territoires porté par les gestionnaires de la réserve est confronté à la position des acteurs locaux, pour qui les ressources naturelles sont également des ressources économiques et sociales, support des pratiques et des cultures locales. Les espaces naturels et institutionnels produits par la réserve reste problématiques, car fondés essentiellement sur une dualité entre "naturel » et « social», conduise à une déterritorialisation ${ }^{12}$ des ressources et des territoires, désormais placés en dehors de l'emprise des sociétés locales, qu'y sont pourtant intimement attachées. La reconstruction d'un lien des sociétés locales avec leur environnement passe par une remise en question, au niveau des gestionnaires et des populations, de la conceptualisation de la dimension «naturelle » du delta.

\section{Bibliographie}

Banu, A. C., RAdulescu, L. (1965). Amenajarea complexa a Deltei pentru punerea in valoare a resourselor naturale. Bucuresti : Editura Stiintifica.

DEVERRE, Chatérine. (2004). Les nouveaux liens sociaux au territoire. Nature, Sciences, Société, 2(12), 172-178.

Di MeO, Guy. (1991). De l'espace subjectif à l'espace objectif : l'itinéraire du labyrinthe, L'Espace géographique, 4, 359-373.

\footnotetext{
${ }^{12}$ Rompre le lien de territorialité entre une société et un territoire (Fournier, 2007).
} 
DUPRE, Lucie. (2007). Les conflits d'environnement : entre sites et réserves. Géographie Économie Société, 2(9), 121-140.

ECHIM, A. (1995). Conditia populatiei din Rezervatia Bisoferei Delta Dunarii. Bucarest : Lex.

FALL, Juliet. (2005). Drawing the line. Nature, Hybridity and Politics in Transboundary Spaces. Abington: Ashgate.

Fournier, Jean-Marc. (2007). Géographie sociale et territoires. De la confusion sémantique à l'utilité sociale?. ESO, 26, 29-35.

Gastescu, P. (1996). The Danube Delta Biosphere Reserve (DDBR). Present state and management. Revue roumaine de géographie. T. 40.

Gastescu, P., SiucA, R. (2006). Delta Dunarri - Rezervatie a Biosferei. Tulcea : Editura Dobrogea.

IUCN. (1992). Conservation Status of the Danube Delta. Environmental Status reports, vol 4.

Mitroi, Veronica., Beaumont, Frédéric. 2011. «Nouvelles frontières et enjeux identitaires dans la région du Delta du Danube ». In Boudreault, P-W. Dressler, W. Aux confins de la nation. Pour une sociologie de la traduction. pp. 201-221.

OST, François. (1995). La Nature hors la loi. L'écologie à l'épreuve du droit. Paris: La Découverte.

World Heritage Nomination - IUCN Summary. Prepared by IUCN (April 1991), based on the original nomination and summary submitted by the Government of Romania. 